EPJ Web of Conferences 73, 01013 (2014)

DOI: $10.1051 /$ epjconf/20147301013

(C) Owned by the authors, published by EDP Sciences, 2014

\title{
Nucleon structure from lattice QCD - recent achievements and perspectives
}

\author{
Constantia Alexandrou ${ }^{1,2, a}$ \\ ${ }^{1}$ Department of Physics, University of Cyprus, PO Box 20537, 1678 Nicosia, Cyprus \\ ${ }^{2}$ Computation-based Science and Technology Research Center, The Cyprus Institute, PO Box 27456, \\ 1645 Nicosia, Cyprus
}

\begin{abstract}
We present recent developments in lattice QCD simulations as applied in the study of hadron structure. We discuss the challenges and perspectives in the evaluation of benchmark quantities such as the nucleon axial charge and the isovector parton momentum fraction, as well as, in the computation of the nucleon $\sigma$-terms, which involve the calculation of disconnected quark loop contributions.
\end{abstract}

\section{Introduction}

There has been spectacular progress in lattice QCD simulations during the past few years, resulting from improvements in algorithms and faster computers. The latest important development is the simulation of the full theory with quark masses tuned to their physical values. Gauge field configurations are now available at near physical pion mass for Wilson-type, staggered and domain wall fermions. This enables us to compute physical quantities directly at the physical point avoiding systematic errors due to the chiral extrapolation, which for baryons are particularly difficult to reliably estimate.

Reproducing the low-lying hadron spectrum has been a milestone for lattice QCD. In Fig. 1 we show the pioneering results for the masses of the octet and decuplet baryons produced by the BMW collaboration using $N_{f}=2+1$ clover fermions [1], as well as, results by the ETM collaboration using $N_{f}=2+1+1$ twisted mass fermions (TMF) [2]. Both collaborations employed simulations with pion masses ranging from about $200 \mathrm{MeV}$ to $500 \mathrm{MeV}$ and extrapolated to the continuum limit using three lattice spacings. In addition, results from the PACS-CS collaboration using $N_{f}=2+1$ clover fermions at one lattice spacing of $a=0.0907$ (13) fm [3] are included. As can be seen, lattice QCD results extrapolated to the continuum limit, taking into account systematic errors as performed by the BMW collaboration are in agreement with the experimental values. In Fig. 1 we also show preliminary results using $N_{f}=2+1+1$ twisted mass fermions for the spin-1/2 and $-3 / 2$ charmed baryons extrapolated to the continuum limit. The mass of the $\Sigma_{c}$ baryon is used to fix the mass of the charm quark obtaining a value that is in agreement with the one extracted from the D-meson mass. Besides the statistical errors shown, systematic errors arising from the tuning of the charm quark mass

\footnotetext{
ae-mail: alexand@ucy.ac.cy
}

This is an Open Access article distributed under the terms of the Creative Commons Attribution License 4.0, which permits unrestricted use, distribution, and reproduction in any medium, provided the original work is properly cited. 


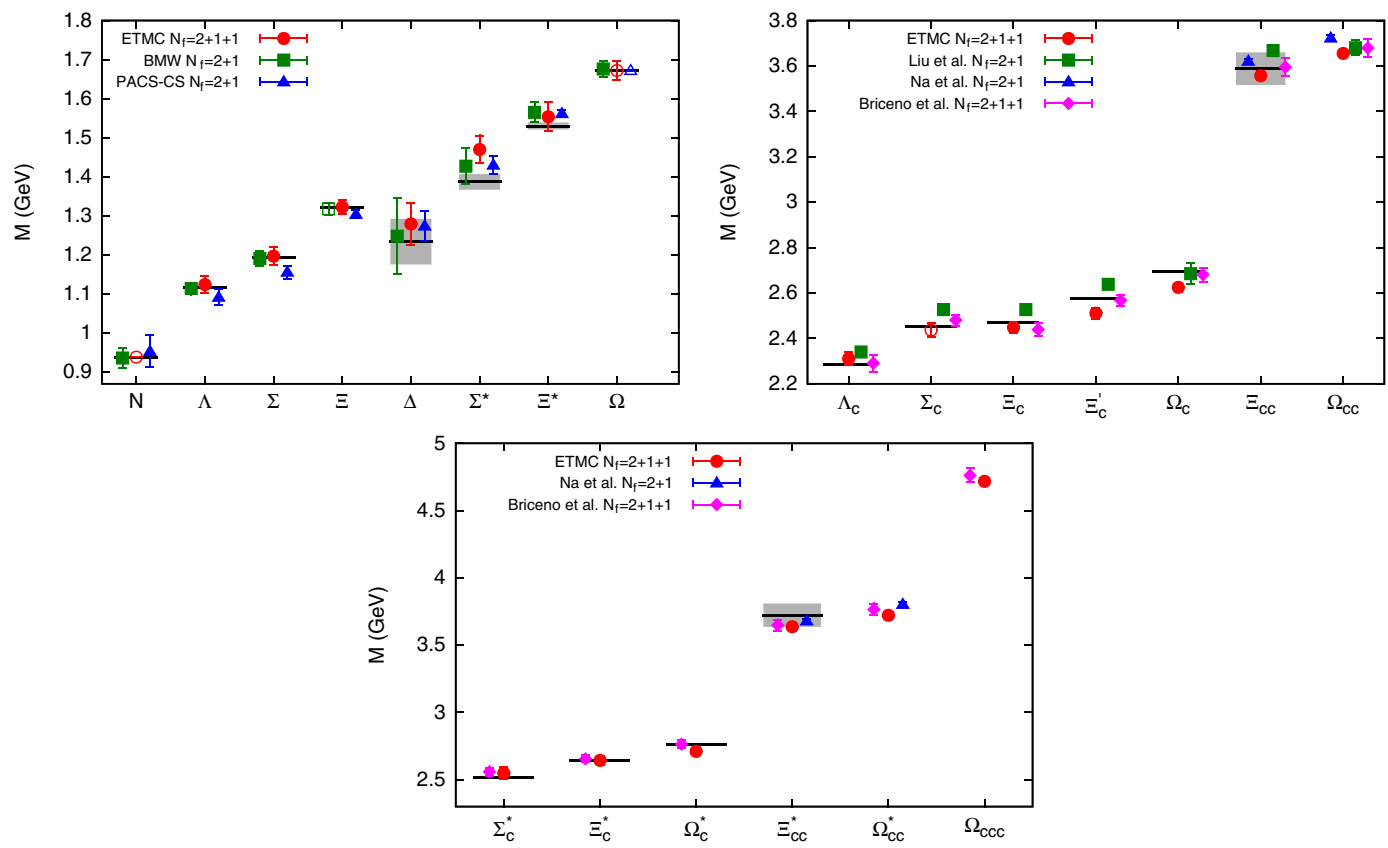

Figure 1. Upper left: the mass of octet and decuplet baryons. Results are by the BMW [1] and the ETM collaborations, extrapolated to the continuum limit and by the PACS-CS collaboration at one lattice spacing [3]. Open symbols show input quantities used to determine the lattice spacing and strange quark mass; upper right: the mass of 1/2-spin charmed baryons using twisted mass fermions and staggered sea quarks [4-6]; lower: The mass of $3 / 2$ spin charmed baryons using twisted mass fermions and staggered sea quarks $[4,6]$. The results by the ETM collaboration are preliminary since only statistical errors are shown.

and the chiral extrapolation are currently being assessed. Results on charmed baryons obtained using staggered gauge field configurations are also shown in Fig. 1. In Ref. [4] $N_{f}=2+1+1$ staggered sea quarks with clover light and strange valence quarks and a relativistic action for the charm quark are employed and the results are extrapolated to the continuum limit. In Refs. [5, 6] $N_{f}=2+1$ staggered sea quarks are used with staggered light and strange [5] or domain wall [6] valence quarks with a relativistic action for the charm quark. These results on the hadron masses demonstrate that the known spectrum including the mass of charmed baryons can be reproduced within lattice QCD thus enabling lattice QCD to provide predictions for the masses of those that have not been measured.

\section{Challenges and perspectives}

In what follows we survey results on benchmark observables, which are still a challenge for lattice QCD and discuss on-going efforts to evaluate systematic errors that may lead to their understanding.

\subsection{Masses of excited states}

While Euclidean correlation functions are very well suited for studies of the lowest hadron state, extracting excited states is harder since they are exponentially suppressed as compared to the ground state. A variational approach, where we enlarge the basis of interpolating fields is the standard approach 

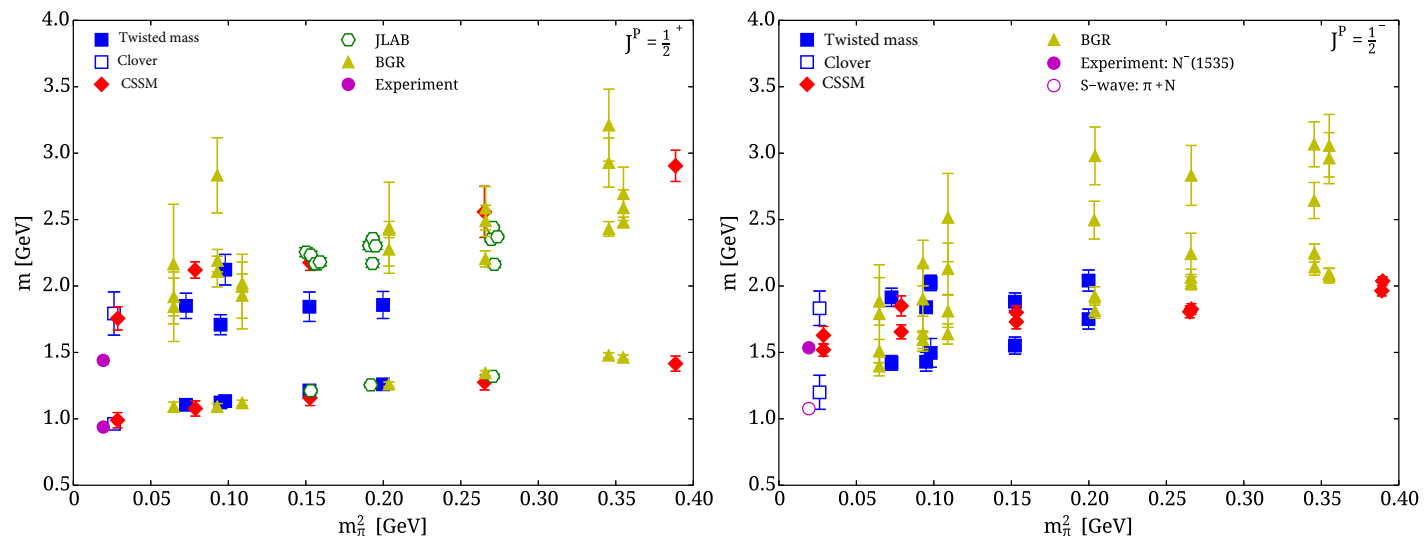

Figure 2. The two lowest nucleon states in the positive (left) and negative (right) parity channels. Results are shown using twisted and clover fermions from Ref. [9], using clover fermions from Refs. [11-13] and using a chirally improved Dirac operator from Ref. [14]. In the negative parity channel the lowest $\pi-N$ scattering state is identified.

employed in order to obtain excited states. We thus consider the correlation matrix

$$
G_{j k}\left(\vec{q}, t_{s}\right)=\sum_{\vec{x}_{s}} e^{-i \vec{x}_{s} \cdot \vec{q}}\left\langle J_{j}\left(\vec{x}_{s}, t_{s}\right) J_{k}^{\dagger}(0)\right\rangle, j, k=1, \ldots N
$$

and solve the generalized eigenvalue problem:

$$
G(t) v_{n}\left(t ; t_{0}\right)=\lambda_{n}\left(t ; t_{0}\right) G\left(t_{0}\right) v_{n}\left(t ; t_{0}\right), \lambda_{n}\left(t ; t_{0}\right)=e^{-E_{n}\left(t-t_{0}\right)}
$$

that yields the $\mathrm{N}$ lowest eigenstates [7]. Large effort has been devoted to construct appropriate bases using lattice symmetries [8] that also includes multi-hadron states. Besides using a suitable variational basis one needs to consider disconnected diagrams as well as develop methods to deal with resonances since most excited states are unstable at the physical pion mass. In this presentation we limit ourselves to examining the first two excited states of the nucleon and in particular the Roper $[9,10]$. In the positive parity channel a linear combination of interpolating fields corresponding to a small and large root mean square radius (rms) produces a wavefunction with a node having potentially a larger overlap with the Roper state. We indeed observe a lowering in the energy of the first excited state when including an interpolating field with a large rms radius. In Fig. 2 we show results on the two lowest states in the positive and negative parity channels. The energy of the $\pi-N$ scattering state is clearly shown in the negative parity channel. However, the results on the Roper and on $S_{11}(1535 \mathrm{MeV})$ generally have larger errors and a systematic study is still required to reach a definite conclusion.

\subsection{Nucleon form factors}

The evaluation of the connected contribution to the three-point function

$$
G^{\mu \nu}\left(\Gamma, \vec{q}, t_{s}, t_{\mathrm{ins}}\right)=\sum_{\vec{x}_{s}, \vec{x}_{\mathrm{ins}}} e^{i \vec{x}_{\mathrm{ins}} \cdot \vec{q}} \Gamma\left\langle J\left(\vec{x}_{s}, t_{s}\right) \mathcal{O}^{\mu \nu}\left(\vec{x}_{\mathrm{ins}}, t_{\mathrm{ins}}\right) \bar{J}\left(\vec{x}_{0}, t_{0}\right)\right\rangle
$$

shown schematically in Fig. 3, is customarily carried out via sequential inversions through the sink. An appropriate ratio of the three-point function with nucleon two-point functions is constructed such that 

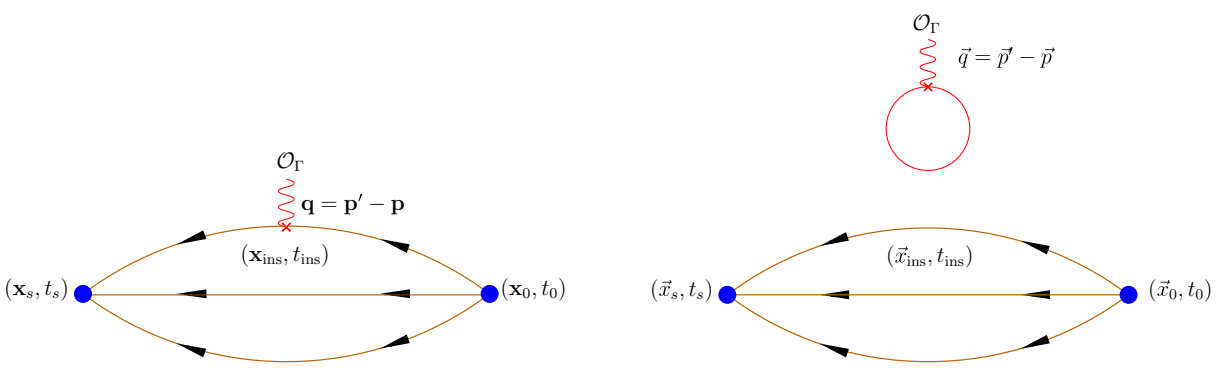

Figure 3. Connected (left) and disconnected (right) contributions to the three-point function.

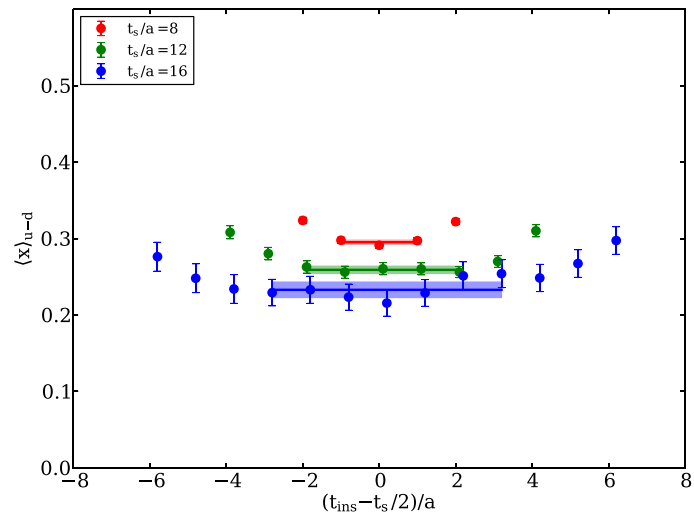

Figure 4. The ratio from which the isovector quark momentum fraction is extracted versus $t_{\text {ins }}$ for sink-source time separations $t_{s}-t_{0}=8 a, 10 a, 12 a$.

the exponential time decay due to the Euclidean time evolution and unknown overlaps cancel. This ratio behaves as

$$
R\left(t_{s}, t_{\text {ins }}, t_{0}\right) \underset{\left(t_{s}-t_{\text {ins }}\right) \Delta \gg 1}{\left(\text { ins }_{0}\right) \Delta \gg 1} \mathcal{M}\left[1+\ldots e^{-\Delta(\mathbf{p})\left(t_{\text {ins }}-t_{0}\right)}+\ldots e^{-\Delta\left(\mathbf{p}^{\prime}\right)\left(t_{s}-t_{\text {ins }}\right)}\right]
$$

where $\mathcal{M}$ the desired matrix element determined from the time-independent value of $R\left(t_{s}, t_{\text {ins }}, t_{0}\right)$ (plateau value) as illustrated in Fig. $4, t_{s}, t_{\text {ins }}$ and $t_{0}$ are the sink, operator insertion and source time-slices and $\Delta(\mathbf{p})$ the energy gap of the lowest state with the first excited state. Lattice results are connected to the measured quantities through a multiplicative renormalization $\mathcal{O}_{\overline{\mathrm{MS}}}(\mu)=Z(\mu, a) \mathcal{O}_{\text {latt }}(a)$ with $Z(\mu, a)$ computed non-perturbatively.

\subsubsection{Axial charge $g_{A}$}

The nucleon matrix element of the axial-vector current $A_{\mu}^{3}=\bar{\psi} \gamma_{\mu} \gamma_{5} \frac{\tau^{3}}{2} \psi(x)$ can be written in terms of two form factors as

$$
\left.\frac{1}{2} \bar{u}_{N}\left(\vec{p}^{\prime}\right)\left[\gamma_{\mu} \gamma_{5} G_{A}\left(q^{2}\right)+\frac{q^{\mu} \gamma_{5}}{2 m} G_{p}\left(q^{2}\right)\right] u_{N}(\vec{p})\right|_{q^{2}=0}
$$

yielding the nucleon axial charge $G_{A}(0) \equiv g_{A} \cdot g_{A}$ is well-measured and has no quark loop contributions and as such it constitutes a benchmark quantity for hadron structure calculations. In Fig. 5 we show 

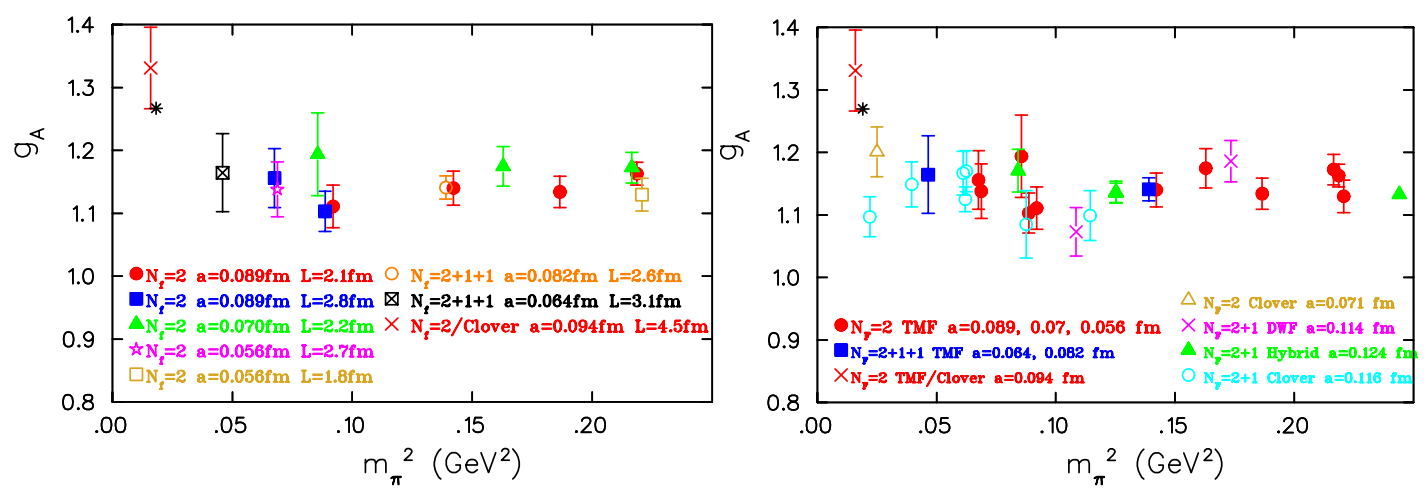

Figure 5. Left: $N_{f}=2$ and $N_{f}=2+1+1$ results with twisted mass fermions for three lattice spacings and different volumes [15]. Right: comparison of lattice results extracted from the plateau value of $R\left(t_{s}, t_{\text {ins }}, t_{0}\right)$ using TMF, $N_{f}=2+1$ clover fermions [16, 17], $N_{f}=2$ clover fermions [18, 19] and a mixed action approach [20].

results using twisted mass fermions and provide a comparison of various lattice results extracted from determining the plateau value $\mathcal{M}$. We note that a number of collaborations are now producing results at or near physical pion mass. We expect that a dedicated study with high statistics, larger volumes and simulations at 3 lattice spacings will be needed in order to finalize these results. Such studies are underway and lattice QCD is poised to resolve the discrepancy on the value of $g_{A}$.

\subsubsection{Momentum fraction}

Another important quantity measured in deep inelastic scattering is the quark momentum fraction $\langle x\rangle_{q}=\int_{0}^{1} d x x[q(x)+\bar{q}(x)]$ as well as the helicity moment $\langle x\rangle_{\Delta q}=\int_{0}^{1} d x x[\Delta q(x)-\Delta \bar{q}(x)]$ where $q(x)=q(x)_{\downarrow}+q(x)_{\uparrow}$ and $\Delta q(x)=q(x)_{\downarrow}-q(x)_{\uparrow}$. In lattice QCD these can be extracted target. by computing the nucleon matrix elements of $\mathcal{O}_{q}^{\mu_{1} \mu_{2}}=\bar{\psi} \gamma^{\left\{\mu_{1}\right.} i \stackrel{\leftrightarrow}{D}{ }^{\left.\mu_{2}\right\}} \psi$ and $\mathcal{O}_{\Delta q}^{\mu_{1} \mu_{2}}=\bar{\psi} \gamma^{\left\{\mu_{1}\right.} \gamma_{5} i \stackrel{\leftrightarrow}{D}{ }^{\left.\mu_{2}\right\}} \psi$. The results on the isovector $\langle x\rangle_{u-d}$ and $\langle x\rangle_{\Delta u-\Delta d}$ in the $\overline{M S}$ scheme at $\mu=2 \mathrm{GeV}$ are summarized in Figs. 6 and 7. As can be seen, results obtained at or near the physical pion mass are converging to the experimental value and, like for the case of $g_{A}$, further studies are expected to resolve the remaining discrepancies.

\section{Disconnected quark loop contributions}

The disconnected quark loop contributions to hadron matrix elements shown schematically in Fig. 3 are notoriously difficult to compute for two reasons: i) they are given by $L\left(x_{\text {ins }}\right)=\operatorname{Tr}\left[\Gamma G\left(x_{\text {ins }} ; x_{\text {ins }}\right)\right]$, which involves the quark propagator from all $\vec{x}_{\text {ins }}$ (i.e. $L^{3}$ more inversions as compared to hadron masses) and ii) they are prone to large gauge noise needing large statistics. To compute such contributions to sufficient accuracy special techniques are utilized that combine i) usage of stochastic noise on all spatial lattice sites, ii) methods that increase statistics at low cost e.g by using low precision inversions (truncated solver method SM) or all-mode-averaging (AMA)), and iii) take advantage of graphics cards (GPUs) by developing special multi-GPU codes [27-30]. As an illustration of such a computation we consider one ensemble of $N_{f}=2+1+1$ twisted mass fermions with lattice spacing $a=0.082 \mathrm{fm}$ and $m_{\pi}=373 \mathrm{Me}$ and perform a high statistics analysis using $\sim 150,000$ measurements for all disconnected contributions to nucleon observables. In Fig. 8 we show the ratio from which the disconnected contributions to $g_{A}^{u+d}$ and $g_{A}^{s}$ are extracted. These quantities determine the quark intrinsic spin $\Delta \Sigma^{q}$. As can be seen, the disconnected contributions are negative and non-zero and must be taken 

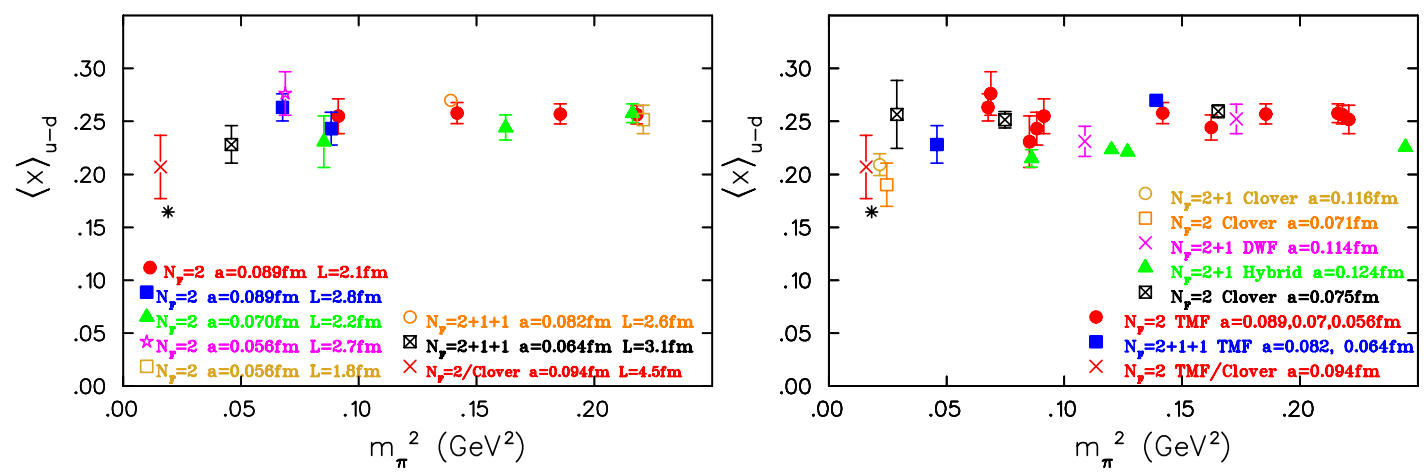

Figure 6. The isovector momentum fraction $\langle x\rangle_{u-d}$ versus $m_{\pi}^{2}$. Results using twisted mass fermions (left) [15] are shown together with a comparison (right) with results using $N_{f}=2$ clover fermions [21, 22], $N_{f}=2+1$ clover fermions [16], $N_{f}=2+1$ domain wall fermions [23] and within a hybrid approach of $N_{f}=2+1$ staggered sea and domain wall valence [24]. The experimental value is taken from Ref. [25].
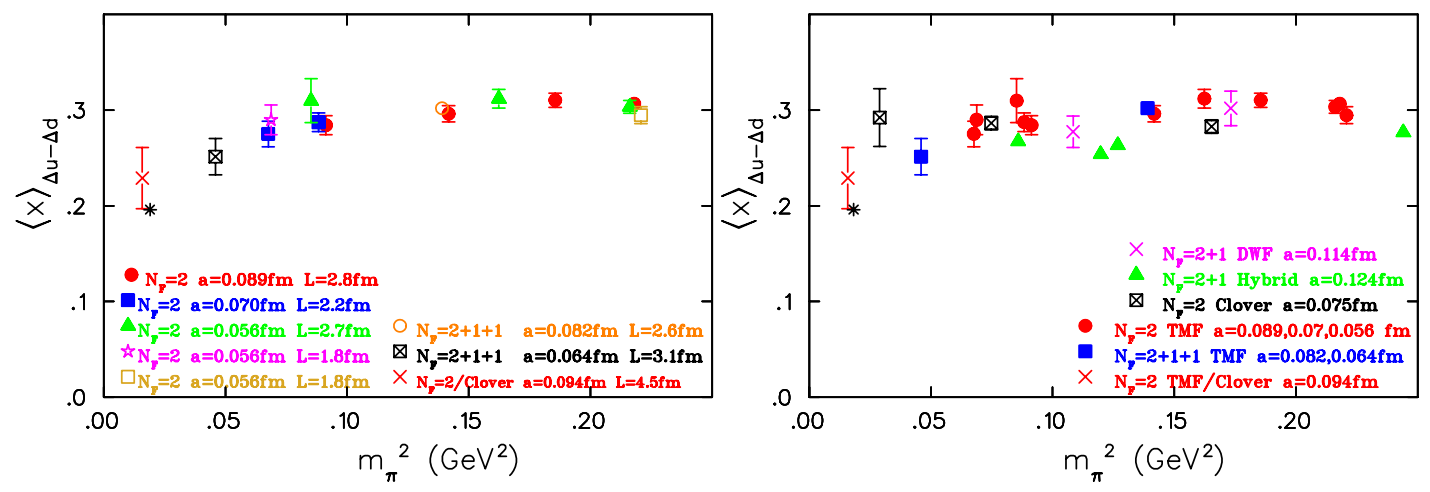

Figure 7. The isovector helicity fraction $\langle x\rangle_{\Delta u-\Delta d}$ versus $m_{\pi}^{2}$. Results using twisted mass fermions (left) [15] are shown together with a comparison (right) with results using $N_{f}=2$ clover fermions [22], $N_{f}=2+1$ domain wall fermions [23] and within a hybrid approach of $N_{f}=2+1$ staggered sea and domain wall valence [24]. The experimental value is taken from Ref. [26].

into account when computing $\Delta \Sigma^{q}$. These results are in agreement with those by QCDSF [31]. The spin in the nucleon satisfies the sum rule $\frac{1}{2}=\sum_{q}\left(\frac{1}{2} \Delta \Sigma^{q}+L^{q}\right)+J^{G}$, where the quark contributions $J^{q}=\frac{1}{2} \Delta \Sigma^{q}+L^{q}$ can be computed from the relation $J^{q}=\frac{1}{2}\left(A_{20}^{q}(0)+B_{20}^{q}(0)\right)$. Furthermore knowing $\Delta \Sigma^{q}=g_{A}^{q}$ we can extract the angular momentum $L^{q}$. In Fig. 9 we show results on $J^{u, d}, \Delta \Sigma^{u+d}$ and $L^{u+d}=J^{u+d}-\Delta \Sigma^{u+d}$ neglecting disconnected contributions except at $m_{\pi}=373 \mathrm{MeV}$ where we also include the result after adding the disconnected contribution that leads to a decrease of $\Delta \Sigma^{u+d}$. What these results show is that the disconnected contributions amount to a $\sim 10 \%$ correction at $m_{\pi} \sim 370 \mathrm{MeV}$ and must be included if we aim at a few percent accuracy. Also we find that $\sum_{q=u, d, s} J^{q} \sim 1 / 4$ so that the question regarding the other $\sim 50 \%$ contribution to the spin of the nucleon still remains open.

\subsection{Nucleon $\sigma$-terms}

The nucleon $\sigma$-term $\sigma_{\pi N} \equiv \frac{\left(m_{u}+m_{d}\right)}{2}\langle N|\bar{u} u+\bar{d} d| N\rangle$ measures the explicit breaking of chiral symmetry and it is an important phenomenological quantity extracted from analysis of low-energy pion-proton scattering data. Its value, determining the Higgs-nucleon coupling, represents the largest uncertainty in 

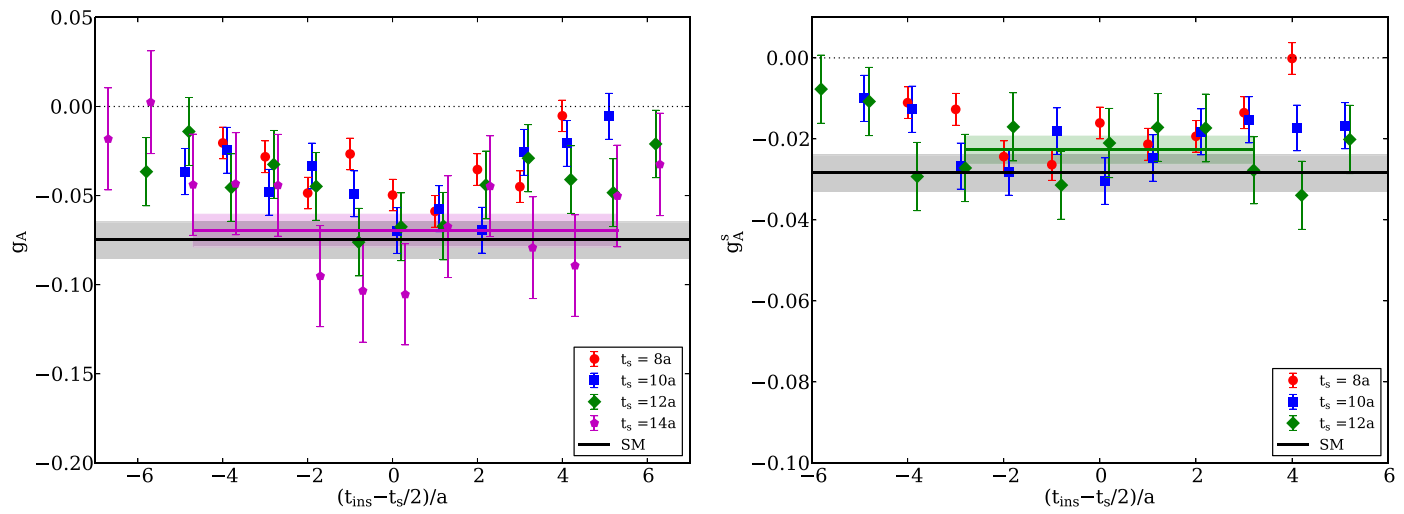

Figure 8. Left: disconnected contribution to the ratio from where the isoscalar $g_{A}^{u+d}$ is extracted; Right: the ratio from which the strange quark contribution $g_{A}^{s}$ is extracted.
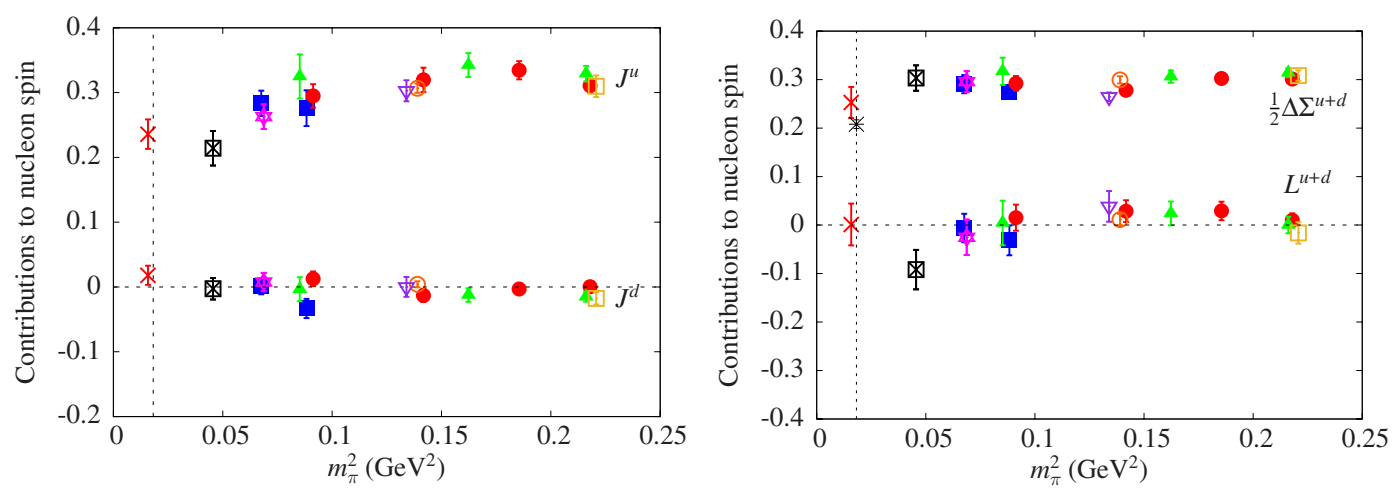

Figure 9. $J^{u}$ and $J^{d}$ (left) and $\Delta \Sigma^{u+d}$ and $L^{u+d}$ (right) as a function of the pion mass. At $m_{\pi}=373 \mathrm{MeV}$ we show the results obtained when we include the disconnected contributions (open inverted purple) triangle using 150,000 measurement as compared to 1200 for the connected part (open red circle).

interpreting experiments for dark matter searches [32]. In lattice QCD it can be computed using the Feynman-Hellman theorem via $\sigma_{q}=m_{q} \frac{\partial m_{N}}{\partial m_{q}}$. A measure for the strange quark content of the nucleon is the ratio $y_{N}=\frac{2\langle N|\bar{s} s| N\rangle}{\langle N|\bar{u} u+\bar{d} d| N\rangle}=1-\frac{\sigma_{0}}{\sigma_{\pi N}}$, where $\sigma_{0}=\langle N|\bar{u} u+\bar{d} d-2 \bar{s} s| N\rangle$ is the flavor non-singlet. A number of groups have used the spectral method to extract the $\sigma$-terms (see e.g. [33]). However, we can now also calculate them directly by computing the three-point functions including the disconnected contributions. In Fig. 10 we show $y_{N}$ as a function of $m_{\pi}$ and extrapolated to the physical pion mass. Due to the cancellation of lattice systematics in the ratio, $y_{N}$ can be computed to a better accuracy than the $\sigma$-terms. We find $y_{N}=0.135(22)(33)(22)(9)$ where the first error is statistical, the second error is estimated using lowest order and next to leading order chiral perturbation theory for the chiral extrapolation, the third is due to excited states contamination and the fourth is an estimate of cut-off effects. Using our value of $y_{N}$ and the phenomenological constrains on $\sigma_{\pi N}$ we can put a bound on the value of $\sigma_{s} \lesssim 250 \mathrm{MeV}$ [34]. 


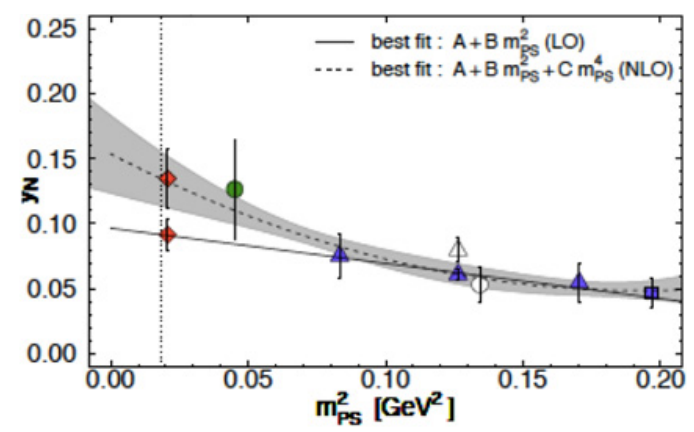

Figure 10. The $y_{N}$-parameter versus $m_{\pi}^{2}$ together with extrapolations to the physical pion mass.

\section{Conclusions}

Nucleon structure is a benchmark for lattice QCD calculations and thus the investigation of $g_{A},\langle x\rangle_{u-d}$, $\langle x\rangle_{\Delta u-\Delta d}$ is considered a central issue. Simulations at the physical pion mass and larger volumes are now becoming available and thus we expect lattice QCD to resolve any remaining discrepancies by using high statistics analysis and careful cross-checks. The evaluation of disconnected quark loop diagrams has also become feasible thus addressing an up to now unknown systematic error. Reproducing the nucleon benchmark quantities will open the way for providing reliable predictions for other hadron observables such axial charges and form factors of hyperons and charmed baryons. Furthermore, appropriate methods to study of excited states, resonances and decays are being developed, with good prospect of providing insight into the structure of hadrons and input that is crucial for experimental searches for new physics.

I would like to thank my collaborators A. Abdel-Rehim, M. Constantinou, V. Drach, K. Hadjiyiannakou, K. Jansen, Ch. Kallidonis, G. Koutsou, Th. Leontiou and A. Vaquero without whom this work would not be possible. I would also like to thank all members of ETMC for a very constructive and enjoyable collaboration and for the many fruitful discussions. Numerical calculations have used HPC resources from John von Neumann-Institute for Computing at the research center in Jülich through the PRACE allocation, and from the Cy- Tera facility of the Cyprus Institute under the project Cy-Tera (NEA YПO $\Delta \mathrm{OMH} / \Sigma T \mathrm{TATH} / 0308 / 31$ ). This work is supported in

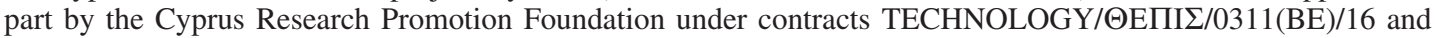
$\Pi$ ПО $\Sigma$ Е $\Lambda$ KY $\Sigma$ H/EMПЕIPO $\Sigma / 0311 / 16$, and the Research Executive Agency of the European Union under Grant Agreement number PITN-GA-2009-238353 (ITN STRONGnet).

\section{References}

[1] S. Durr et al., Science 322, 1224 (2008)

[2] C. Alexandrou, V. Drach, K. Jansen, C. Kallidons et al. (ETM Collaboration) (2014), in preparation

[3] S. Aoki et al. (PACS-CS), Phys. Rev. D 79, 034503 (2009), 0807.1661

[4] R.A. Briceno, H.W. Lin, D.R. Bolton, Phys. Rev. D 86, 094504 (2012), 1207.3536

[5] H. Na, S. Gottlieb, PoS LATTICE2008, 119 (2008), 0812.1235

[6] L. Liu, H.W. Lin, K. Orginos, A. Walker-Loud, Phys. Rev. D 81, 094505 (2010), 0909. 3294

[7] M. Lüscher, U. Wolff, Nucl. Phys. B 339, 222 (1990) 
[8] C. Morningstar, J. Bulava, B. Fahy, J. Foley, Y. Jhang et al., Phys. Rev. D 88, 014511 (2013), 1303.6816

[9] C. Alexandrou, T. Korzec, G. Koutsou, T. Leontiou (2013), 1302.4410

[10] C. Alexandrou, M. Constantinou, V. Drach, K. Hatziyiannakou, K. Jansen et al. (2013), 1303.6818

[11] M. Mahbub, W. Kamleh, D. Leinweber, P. Moran, A. Williams, AIP Conf. Proc. 1441, 293 (2012)

[12] M.S. Mahbub, W. Kamleh, D.B. Leinweber, P.J. Moran, A.G. Williams, Phys. Rev. D 87, 094506 (2013), 1302.2987

[13] R.G. Edwards et al., Phys. Rev. D 84, 074508 (2011), 1104.5152

[14] G.P. Engel, C. Lang, D. Mohler, A. Schäfer (BGR), Phys. Rev. D 87, 074504 (2013), 1301.4318

[15] C. Alexandrou, M. Constantinou, V. Drach, K. Jansen, C. Kallidonis et al. (2013), 1312. 2874

[16] J. Green, M. Engelhardt, S. Krieg, J. Negele, A. Pochinsky et al. (2012), 1209.1687

[17] B.J. Owen, J. Dragos, W. Kamleh, D.B. Leinweber, M.S. Mahbub et al., Phys. Lett. B 723, 217 (2013), 1212.4668

[18] R. Horsley, Y. Nakamura, A. Nobile, P. Rakow, G. Schierholz et al. (2013), 1302. 2233

[19] S. Capitani, M. Della Morte, G. von Hippel, B. Jager, A. Juttner et al., Phys. Rev. D 86, 074502 (2012), 1205.0180

[20] T. Bhattacharya, S.D. Cohen, R. Gupta, A. Joseph, H.W. Lin (2013), 1306.5435

[21] G.S. Bali, S. Collins, M. Deka, B. Glassle, M. Gockeler et al., Phys. Rev. D 86, 054504 (2012), 1207.1110

[22] D. Pleiter et al. (QCDSF/UKQCD Collaboration), PoS LATTICE2010, 153 (2010), 1101. 2326

[23] Y. Aoki, T. Blum, H.W. Lin, S. Ohta, S. Sasaki et al., Phys. Rev. D 82, 014501 (2010), 1003. 3387

[24] J.D. Bratt et al. (LHPC), Phys. Rev. D 82, 094502 (2010), 1001. 3620

[25] S. Alekhin, J. Blumlein, S. Moch, Phys. Rev. D 86, 054009 (2012), 1202. 2281

[26] J. Blumlein, H. Bottcher, Nucl. Phys. B 841, 205 (2010), 1005.3113

[27] C. Alexandrou, K. Hadjiyiannakou, G. Koutsou, A. O'Cais, A. Strelchenko, Comput. Phys. Commun. 183, 1215 (2012), 1108.2473

[28] C. Alexandrou, M. Constantinou, V. Drach, K. Hadjiyiannakou, K. Jansen et al. (2013), 1309.2256

[29] C. Alexandrou, V. Drach, K. Jansen, G. Koutsou, A. Vaquero (2014), 1401.6749

[30] C. Alexandrou, K. Hadjiyiannakou, G. Koutsou, A. Strelchenko, A. Vaquero (2014), 1401.6750

[31] G.S. Bali et al. (QCDSF Collaboration), Phys. Rev. Lett. 108, 222001 (2012), 1112.3354

[32] J.R. Ellis, K.A. Olive, C. Savage, Phys. Rev. D 77, 065026 (2008), 0801.3656

[33] R. Young, PoS LATTICE2012, 014 (2012), 1301.1765

[34] C. Alexandrou, M. Constantinou, S. Dinter, V. Drach, K. Hadjiyiannakou et al. (2013), 1309.7768 